ARTICLE

https://doi.org/10.1038/s41467-019-12437-6

\title{
In situ analysis of catalyst composition during gold catalyzed GaAs nanowire growth
}

\author{
Carina B. Maliakkal (1) 1,2*, Daniel Jacobsson (1) 1,3, Marcus Tornberg (1) 1,2, Axel R. Persson (1) 1,3, \\ Jonas Johansson (10 1,2, Reine Wallenberg ${ }^{1,3}$ \& Kimberly A. Dick (D) 1,2,3
}

Semiconductor nanowires offer the opportunity to incorporate novel structures and functionality into electronic and optoelectronic devices. A clear understanding of the nanowire growth mechanism is essential for well-controlled growth of structures with desired properties, but the understanding is currently limited by a lack of empirical measurements of important parameters during growth, such as catalyst particle composition. However, this is difficult to accurately determine by investigating post-growth. We report direct in situ measurement of the catalyst composition during nanowire growth for the first time. We study Au-seeded GaAs nanowires inside an electron microscope as they grow and measure the catalyst composition using X-ray energy dispersive spectroscopy. The Ga content in the catalyst during growth increases with both temperature and Ga precursor flux.

\footnotetext{
${ }^{1}$ NanoLund, Lund University, 22100 Lund, Sweden. ${ }^{2}$ Solid State Physics, Lund University, Box 118, 22100 Lund, Sweden. ${ }^{3}$ National Center for High Resolution Electron Microscopy and Centre for Analysis and Synthesis, Lund University, Box 124, 22100 Lund, Sweden. *email: carina_babu.maliakkal@ftf.Ith.se
} 
$\mathrm{N}$ anowire growth by the vapor-liquid-solid (VLS) method is an important technique for producing well-controlled nanocrystals suitable for quantum components. For III-V semiconductors, an important material system for future technologies within electronics, solid-state lighting and quantum processing, VLS growth enables the fabrication of, for example, lattice-mismatched heterostructures ${ }^{1-3}$, metastable crystal phases and crystal-phase tuning ${ }^{4-7}$, and unusual ternary alloys ${ }^{8,9}$. VLS growth has been well-studied for over two decades, and extensive theoretical efforts exist to explain the growth process itself ${ }^{10-14}$, the observed trends with experimental parameters ${ }^{15-18}$, and the existence of metastable structures ${ }^{19-21}$. However, validation of theoretical predictions remains extremely difficult due to the large number of variable material properties and accessible experimental parameters, and the subsequent variance in reported experimental trends. In addition, many of the important fundamental parameters, such as surface and interface energies for relevant growth conditions, are unknown ${ }^{22,23}$. Consequently, there are a wide range of competing and complementary models that can explain observations, such as crystal phase trends ${ }^{6,24-26}$ and diameter-growth rate dependencies ${ }^{27-32}$.

The use of in situ characterization methods to gain direct insights into semiconductor nanowire growth in real time is one of the most effective ways to refine theoretical explanations and predictions, and in turn to better understand the conditions needed to design these materials with high control ${ }^{33}$. Examples of previous in situ studies include X-ray diffraction to understand phase and structural evolution ${ }^{34-36}$, infrared spectroscopy to correlate surface chemistry during growth with resulting nanowire morphology $y^{37-39}$, reflectance high-energy electron diffraction to follow nucleation and structural changes ${ }^{40-42}$, optical reflectometry to monitor growth rate evolution ${ }^{43,44}$, and mass spectrometry to study nucleation ${ }^{45}$. In addition, in situ scanning electron microscopy has been used to directly follow nanowire growth and morphology, and combined with Auger electron spectroscopy to track surface chemistry ${ }^{46}$. Finally, in situ transmission electron microscopy (TEM) has proven to be one of the most powerful ways to gain insight into nanowire growth in a directly interpretable way. Importantly, the information provided by this method is local, meaning that the nanowire, the growthenabling liquid droplet and the interface between them can be visually identified and independently studied. This method has led to numerous significant breakthroughs in nanowire growth such as vapor-solid-solid growth ${ }^{47,48}$, corner truncation ${ }^{49,50}$, and step flow ${ }^{48}$. In situ TEM studies have been particularly beneficial for understanding bilayer growth kinetics ${ }^{51}$, crystal-phase switching $^{52}$, triple-phase-line nucleation ${ }^{53}$, and double-bilayer growth $^{54}$ in III-V nanowires.

One essential aspect that remains to be investigated is the local composition of the nanostructure during growth. This is necessary to understand composition evolution in, for instance, heterostructure and ternary nanowires, but even more importantly, for understanding the composition of the liquid metal droplet as a function of growth parameters and how this is correlated with the resulting nanowire properties ${ }^{55}$. The composition of the catalyst particle is a pivotal factor that determines its thermodynamic parameters, such as vapor pressure, chemical potential, and surface energies, which in turn decide the nanowire structure, growth rate, composition, etc. ${ }^{56-58}$. So far, the composition of the catalyst particles have been measured post growth and was shown to depend on the conditions used for terminating the growth $^{4,59,60}$ (more details in Supplementary Discussion 1), implying that post-growth composition of the particle is different from its composition during growth. To our knowledge, there has been no direct in situ measurement of catalyst composition during nanowire growth. An indirect estimation of the $\mathrm{Au}-\mathrm{Ga}$ catalyst composition during GaAs nanowire growth has been reported by comparing the dimensions of the starting Au seeds particles and the catalyst during growth by assuming that the seed material does not diffuse out of the catalyst particle ${ }^{52}$.

In this article, we report direct in situ measurement of catalyst composition during nanowire growth. We use in situ X-ray energy dispersive spectroscopy (XEDS) combined with in situ TEM to investigate the composition of the metal droplet as a function of growth parameters for $\mathrm{Au}$-seeded $\mathrm{GaAs}$ nanowires grown by the VLS method. We show that the droplet consists of a significant quantity of $\mathrm{Ga}$ for all growth conditions, which increases with temperature for constant precursor flow. We do not observe any As significantly above the detection limit of the XEDS technique. Using calculated ternary-phase diagrams, we show that a lower bound on the As content can, however, be estimated. We also observe that the Ga content of the droplet for a given temperature is relatively independent of the ratio of V/III precursor species, so long as this ratio is above a certain threshold. Below this threshold, the Ga content increases strongly with decreasing V/III ratio, accompanied by a volume increase in the droplet. We show that the droplet volume scales with the Ga content, validating earlier works that used volume as an estimate of $\mathrm{Ga}^{52}$. The trend with V/III ratio is understood to correlate with a gradual transition toward the so-called "arsenic-limited" growth, whereby the droplet initially swells up but eventually stabilizes $^{61}$. The results demonstrate that in situ XEDS is a useful and direct way to gain important insight and information on nanowire growth in real time, and will be similarly appropriate for other types of processes occurring at similar temperatures and overall gas pressures. Finally, the measurements of the droplet composition as a function of growth parameters will provide important inputs for validation and modification of theoretical models describing nanowire growth ${ }^{14,55,62}$.

\section{Results and discussion}

Growth of GaAs nanowires and in situ measurements. Au nanoparticles deposited on a silicon nitride-based heating grid were used to seed the nanowire growth. Nanowires were grown inside a Hitachi HF3300S environmental TEM integrated with a custom metal organic chemical vapor deposition (MOCVD) system. Trimethylgallium (TMGa) and arsine $\left(\mathrm{AsH}_{3}\right)$, which are the most common precursor gases in MOCVD growth of GaAs, were used for this study. The chemical composition of the catalyst was studied by XEDS as a function of temperature and the ratio of precursor fluxes, which are two very important parameters in typical MOCVD growth. Please refer to the Methods section for more technical details.

XEDS of catalyst measured in situ. The catalyst composition was measured using XEDS in situ as the GaAs nanowire grows. An example XEDS spectrum is shown in Fig. 1b from the nanowire shown in Fig. 1a. Clear signals from $\mathrm{Ga}$ and $\mathrm{Au}$ in the catalyst are observed for all spectra, along with a strong Si signal arising from the $\mathrm{SiN}_{x}$ grid on which the nanowires are growing, and system artefacts such as $\mathrm{Cu}$ arising from microscope components. (The same spectrum but with a broader $x$-axis range is shown in Supplementary Fig. 1.) Quantification of the XEDS spectrum in Fig. $1 \mathrm{~b}$, measured at $440{ }^{\circ} \mathrm{C}$, gives a Ga:Au atomic ratio of $\sim 30: 70$ (assuming only $\mathrm{Ga}$ and $\mathrm{Au}$ are present). Some spectra also show small features that could be due to As, amounting to a maximum of $\sim 1-3$ atomic \% (with normalization $\mathrm{Au}+\mathrm{Ga}+\mathrm{As}=100 \%$ ). However, the peak is too small to be conclusively attributed to As from the catalyst and quantified. Scattering into the GaAs nanowire could easily give rise to this signal (further information is available in the Methods section), in which case the Ga 
concentration mentioned throughout the draft could be overestimated by a couple of percent. Though any As within the catalyst is too low to be directly quantified, it is certainly below 3\%. (So in the following sections, we quote $\mathrm{Ga} \%$ and $\mathrm{Au} \%$ measured in the catalyst after renormalizing such that $\mathrm{Au}+\mathrm{Ga}$ $=100 \%$, unless otherwise specified.) Our observation of very low concentration of As in the catalyst during growth is consistent with theoretical calculations; for instance, Glas et al. calculate the As content to be $\sim 1 \%$ (depending on radius and contact angle) ${ }^{63}$. Mårtensson et al. predicted As content to be roughly in the range $0.01-1 \%$ depending on the growth conditions ${ }^{64}$. Post-growth XEDS reports have also claimed As to be below detection limits ${ }^{60}$.

a

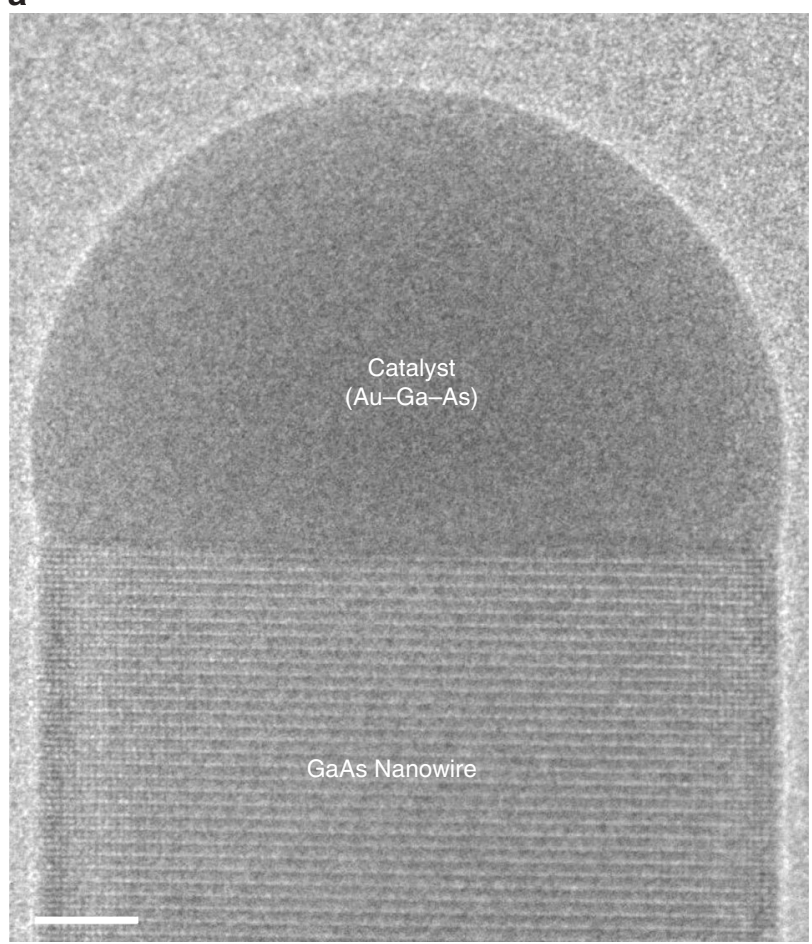

b

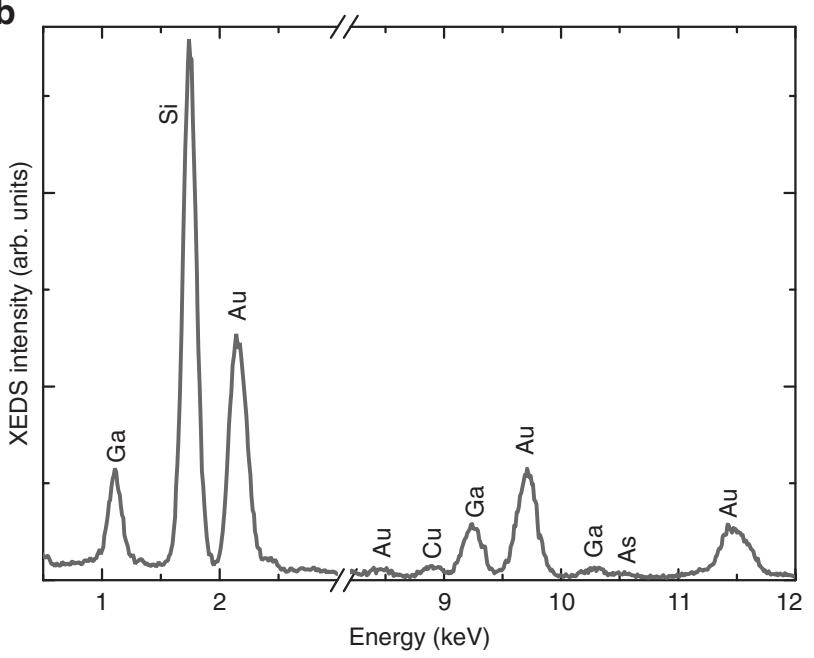

Fig. 1 In situ Au-seeded GaAs nanowire growth. a TEM image of a GaAs nanowire growing inside the TEM on a $\mathrm{SiN}_{x}$ grid at $440{ }^{\circ} \mathrm{C}$, and $\mathrm{V} / \mathrm{III}$ ratio of 3780 . Scale bar indicates $5 \mathrm{~nm}$. b XEDS spectrum of the catalyst particle at the same conditions measured in situ during its growth. The atomic species giving rise to the different peaks are indicated in the plot
Catalyst composition as a function of temperature. The composition of a catalyst particle during nanowire growth was measured as a function of temperature and is shown in Fig. 2. During this experiment, the Ga precursor flow was set to be relatively low $(\mathrm{V} / \mathrm{III}=3780$; the relevance of this choice will be discussed later $)$.
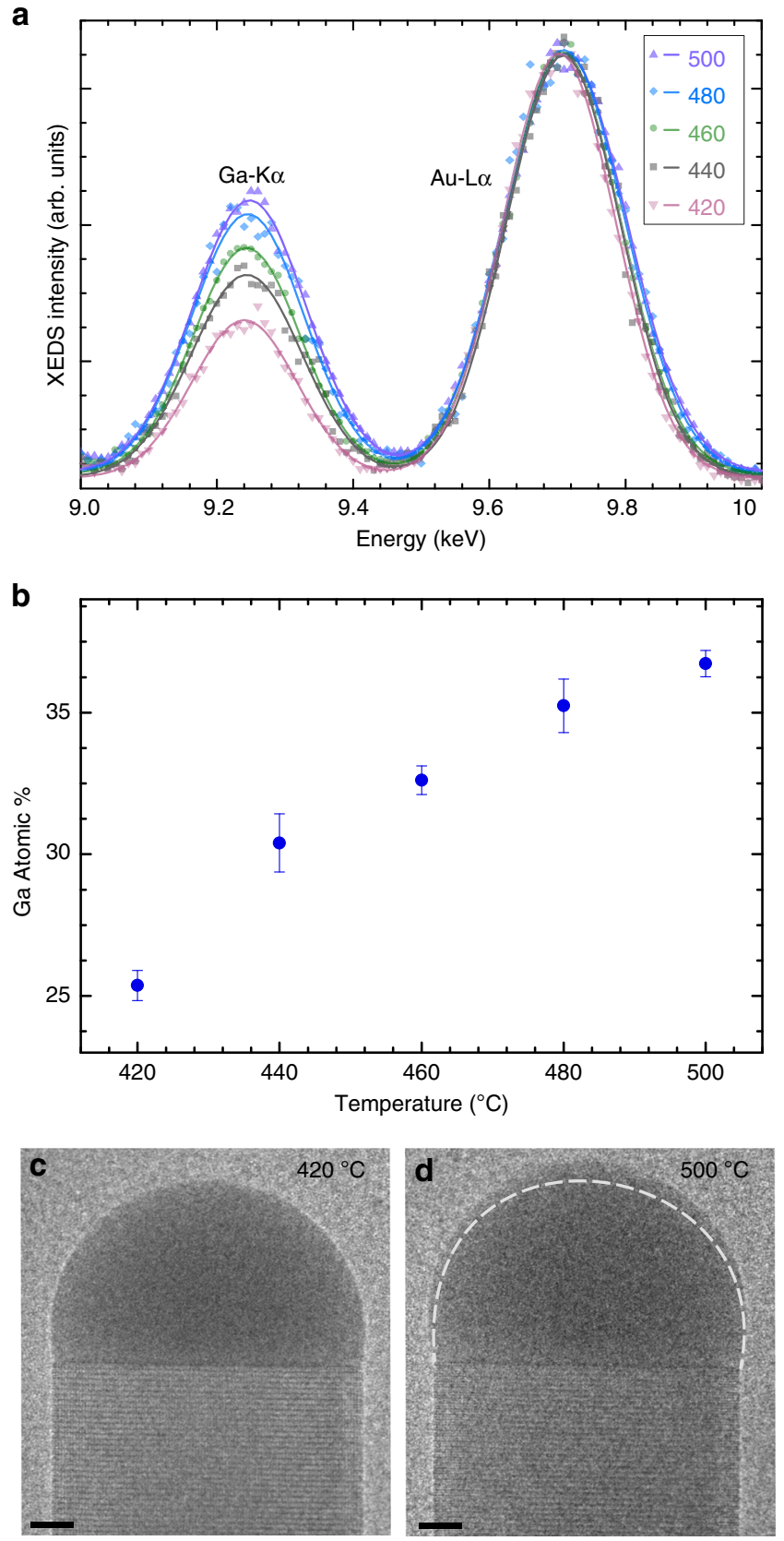

Fig. 2 Catalyst composition as a function of temperature. a The Ga-K $\alpha$ and $\mathrm{Au}-\mathrm{L} \alpha$ peaks in XEDS spectra measured at different temperatures (in ${ }^{\circ} \mathrm{C}$ ). These spectra are normalized with respect to the Au-L $\alpha$ peak. The Ga peak intensity increases relative to the Au peak with increasing temperature. b The atomic percentage of $\mathrm{Ga}$ in one catalyst particle measured as a function of temperature at constant V/III ratio. Error bars show SD in the XEDS quantification. With increasing temperature, the catalyst stabilizes with more Ga resulting in larger catalyst particle as seen in panels $\mathbf{c}$ and d. TEM images of the catalyst at $420^{\circ} \mathrm{C}$ (c) and $500^{\circ} \mathrm{C}$ (d). At $500^{\circ} \mathrm{C}$, the catalyst is larger than at $420^{\circ} \mathrm{C}$. The outline of the catalyst at $420^{\circ} \mathrm{C}$ is depicted on top of the TEM image at $500^{\circ} \mathrm{C}$ (d) by a white dashed line. Scale bars in panels $\mathbf{c}$ and $\mathbf{d}$ indicate $5 \mathrm{~nm}$ 
More details of the experiment can be found in the Methods section. The Ga content in the catalyst increases with temperature as can be observed in the XEDS spectra normalized to the Au-L $\alpha$ peak (Fig. 2a). Quantification of the XEDS spectra shows that when the temperature was increased monotonically from 440 to $500{ }^{\circ} \mathrm{C}$, the $\mathrm{Ga}$ content increased from 30 to 36 atomic \% (with $\mathrm{Au}+\mathrm{Ga}=100 \%$ ) (Fig. 2b). The temperature was then decreased to $420^{\circ} \mathrm{C}$, after which the $\mathrm{Ga}$ content decreased. A small but measurable change in the volume of the catalyst droplet at different temperatures was observed, as illustrated in Fig. $2 c$, indicating that the composition change is primarily due to an increase in $\mathrm{Ga}$ rather than a loss of $\mathrm{Au}$ atoms. (The correlation between $\mathrm{Ga}$ concentration and catalyst volume is discussed in more detail in Supplementary Discussion 9.) A separate experiment performed on a different nanowire, at a relatively lower temperature range than shown in Fig. 2 is given in Supplementary Fig. 3; which also shows an increase in the Ga content with temperature. The effect of temperature on growth is multifold-precursor decomposition (of both $\mathrm{TMGa}$ and $\mathrm{AsH}_{3}$ ), Ga surface diffusion on the nanowire sidewalls, As evaporation rate, flow patterns in the growth cell, surface energies etc. depend on temperature. So a straightforward explanation of the observed trend is difficult.

The TMGa supply for this temperature series experiment was in a regime where the $\mathrm{Ga}$ content is rather insensitive to the $\mathrm{Ga}$ flow at $420^{\circ} \mathrm{C}$ (will be explained later), indicating that it is not kinetically controlled. We therefore turn to thermodynamic considerations to understand the observations. Nanowire growth is understood to occur once the growth species becomes supersaturated in the catalyst ${ }^{65,66}$. Generally the growth is described as a cyclic process where a refilling step alternates with a layer growth step, and so the Ga and As concentrations oscillate between a maximum (just before a layer starts to grow) and a minimum (when a layer finishes). The lowest values for the minimum concentrations correspond to the equilibrium concentrations of these species in the liquid catalyst, termed the thermodynamic reference state of the system ${ }^{66}$. Ternary-phase diagrams provide a visualization of this reference state. The maximum allowed As and $\mathrm{Ga}$ content for a liquid $\mathrm{Au}-\mathrm{Ga}-\mathrm{As}$ alloy in thermodynamic equilibrium is given by the liquidus line (blue line in Fig. 3a) in the phase diagram. Since the catalyst must be supersaturated during growth, the composition must always be (slightly) to the right of the liquidus line, allowing us to estimate the minimum As concentration based on our measured $\mathrm{Ga}$ concentrations. Liquidus lines for a few representative As concentrations are shown in Fig. 3b. Using the measured Ga \% at $420^{\circ} \mathrm{C}$ and imposing the necessity of supersaturation, we can estimate the minimum As \% in the catalyst to be $\sim 0.01 \%$.

It is worth noting that the Ga \% measured by XEDS while the nanowire grows is the average concentration over a period of several minutes (typically $4 \mathrm{~min}$ ), rather than the minimum $\mathrm{Ga}$ concentration. For a slightly lower Ga concentration, As would be slightly higher, and so the minimum As \% of $\sim 0.01 \%$ is a conservative order-of-magnitude estimate: the real value is expected to be slightly higher. An upper bound on the As concentration cannot be deduced from thermodynamic considerations, but is expected to be on the order of $1 \%$ based on the in situ XEDS measurements discussed above. Interestingly, the number of excess atoms required to form an entire GaAs bilayer would correspond to $\sim 1 \%$ in the catalyst droplet for the catalyst/ nanowire dimensions discussed here, meaning that there are never enough atoms stored in the droplet at one time for an entire layer to form at once. For the parameter ranges used in this article, it is indeed observed that the growth of each layer is not instantaneous ${ }^{67}$. (Further details on the step-flow propagation are discussed as a separate study in ref. ${ }^{67}$, including experiments at conditions similar to that being discussed here.)
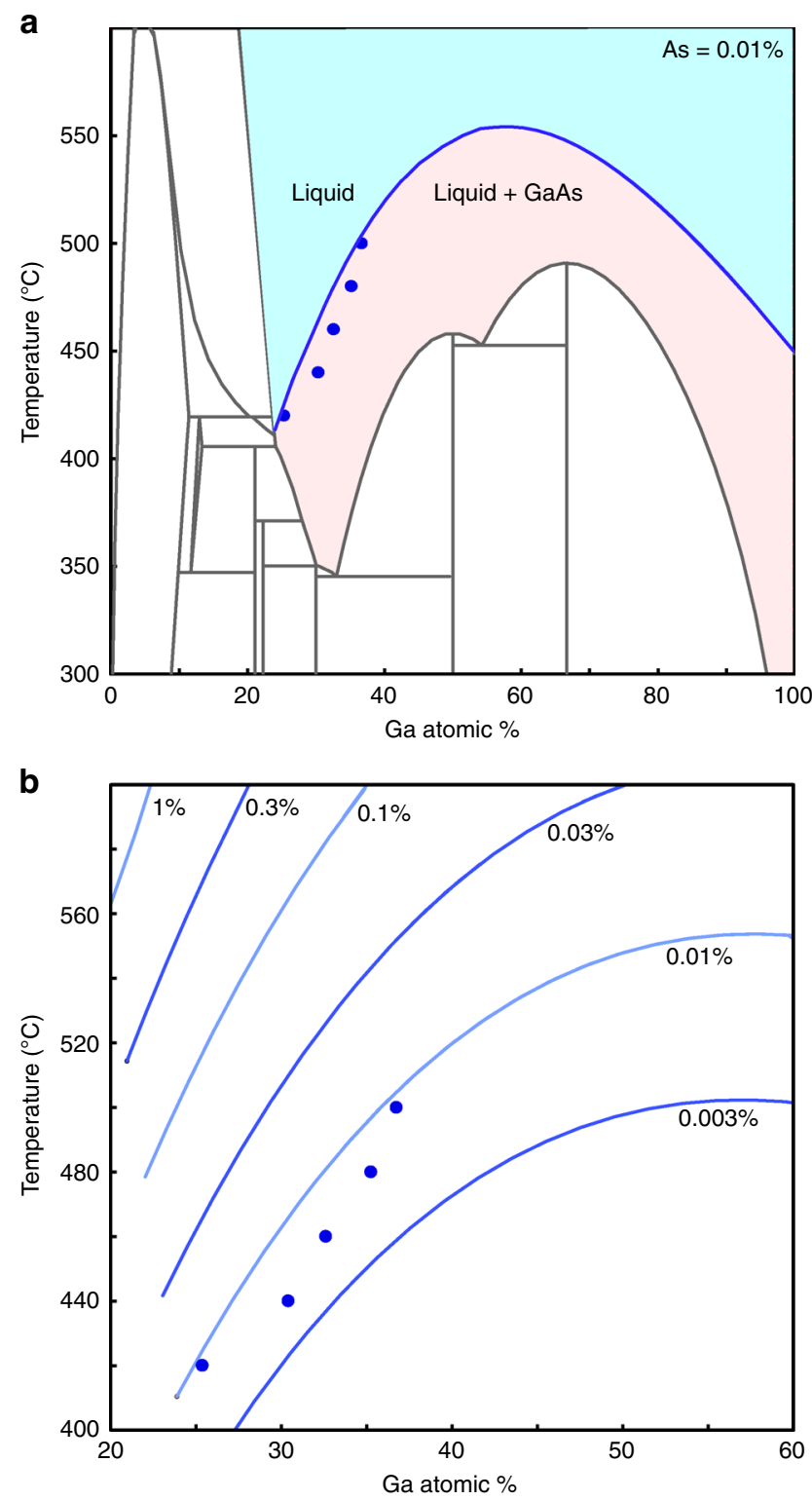

Fig. 3 Comparison of measured Ga with constant As phase diagram sections. a Au ${ }_{99.99 \%} \mathrm{As}_{0.01 \%}-\mathrm{Ga}_{99.99 \%} \mathrm{As}_{0.01 \%}$ phase diagram (or projection of the Au-Ga-As phase diagram at a fixed As content of $0.01 \%$ ). The choice of this concentration of As was such that the measured Ga percentage lies in the supersaturation regime (i.e., right side or below the liquidus line shown by the blue curve). Blue dots are the experimentally measured Ga content. b Liquidus line calculated for different As concentrations. The As concentration is labeled on each liquidus

Catalyst composition as a function of precursor flux. V/III ratio i.e., the ratio of the group $\mathrm{V}$ precursor flux $\left(\mathrm{AsH}_{3}\right.$ here) to the group III precursor (TMGa here), is a very important parameter for the growth of III-V semiconductors. We now discuss the change of catalyst composition as a function of V/III ratio, at a fixed temperature of $420^{\circ} \mathrm{C}$ measured on another nanowire (Fig. 4a, b). (Please see the Methods section for details on how the V/III ratio is measured in this experiment). We had set the $\mathrm{AsH}_{3}$ flow to be fixed and changed only the TMGa flow in this experiment. When the TMGa flow was stopped the nanowire was neither growing nor etching; the Ga content in the catalyst was then measured to be $27 \%$ by XEDS. (At elevated temperatures 
GaAs gets slowly etched by the Au-based catalyst if the precursors are not supplied appropriately ${ }^{4,68}$ ). The Ga concentration in the catalyst then increases monotonically with increasing $\mathrm{Ga}$

a

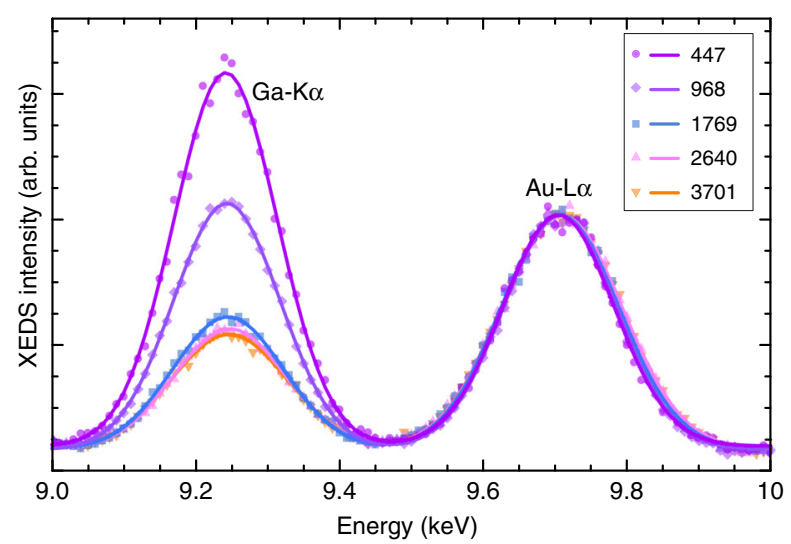

b

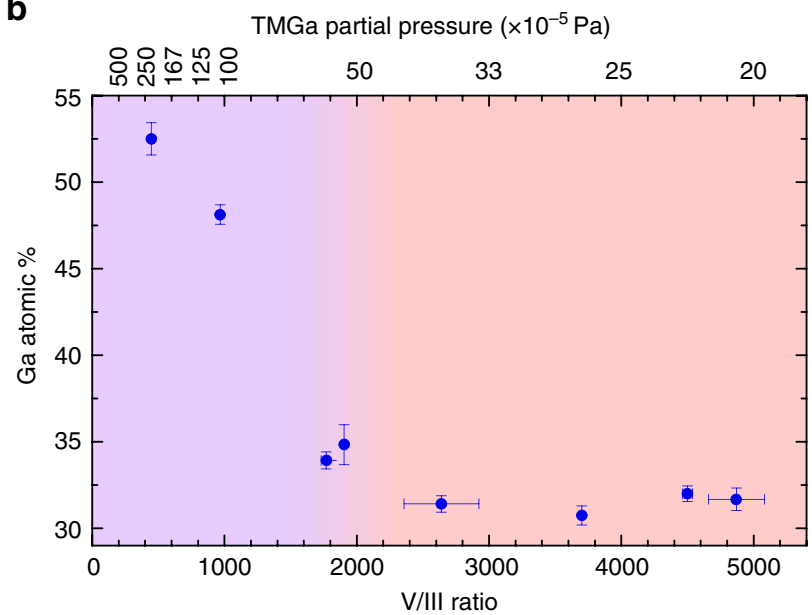

C

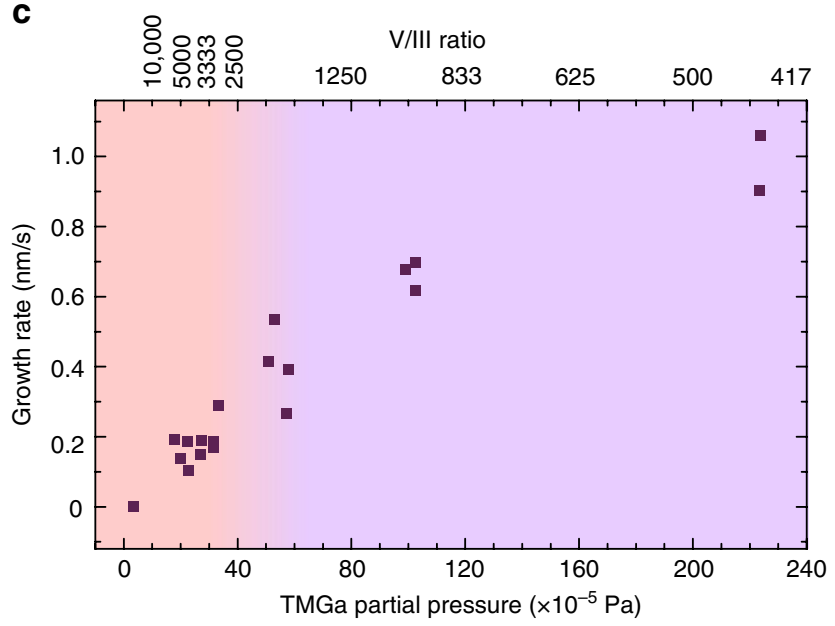

Fig. 4 Catalyst composition and growth rate as a function of V/III ratio. a The $\mathrm{Ga}-\mathrm{K} \alpha$ and $\mathrm{Au}-\mathrm{L} \alpha$ peaks in XEDS spectra measured at different $\mathrm{V} / \mathrm{III}$ ratios. These spectra are normalized with respect to the Au-L $\alpha$ peak. The Ga peak intensity relative to the Au peak increases with decreasing V/III ratio. b The Ga content in one catalyst particle measured for varying V/III ratio. Error bars show SD in the XEDS quantification. c Growth rate measured from the videos plotted as a function of TMGa partial pressure. The background gradient color in $\mathbf{b}, \mathbf{c}$ is such that purple indicates high TMGa regime while peach indicates low TMGa regime. Note that the top axis in $\mathbf{b}, \mathbf{c}$ is nonlinear precursor flux. At high V/III ratios (or low TMGa) a small increase of TMGa does not change the Ga concentration much; in fact, the Ga concentration is effectively constant within the resolution limit of the XEDS measurement. Since the Ga content is in a steady state (between incoming $\mathrm{Ga}$, controlled by the TMGa flow, and outgoing Ga, primarily controlled by the nanowire growth), this result suggests that the nanowire growth is mainly limited by the TMGa flow in this regime. This is the regime that we used for comparison with phase diagrams. At low V/III ratios i.e., below $\sim 2000$ (Fig. $4 \mathrm{~b}$ ), the Ga concentration increases rapidly with increasing TMGa. (A similar increase of $\mathrm{Ga}$ content at lower V/III ratio was observed at $500{ }^{\circ} \mathrm{C}$ by changing Ga flow. This is shown in Supplementary Fig. 5. An analogous trend was also observed when V/III ratio is varied by changing the $\mathrm{AsH}_{3}$ flow instead, as shown in Supplementary Fig. 6). This trend in the measured composition by XEDS is accompanied by a large, clearly visible increase in the size of the catalyst droplet. Similar swelling of the catalyst at low V/III has previously been observed both ex situ ${ }^{69,70}$ and in situ ${ }^{51,52}$, and has also been predicted theoretically ${ }^{71}$. Previous reports have associated this effect with a transition to a V-limited regime where the Ga supply is effectively higher than the As supply, and so excess Ga accumulates in the droplet before reaching a new quasi-steady-state composition $^{51,52}$.

The growth rate of the nanowire as a function of TMGa partial pressure (and V/III ratio) is shown in Fig. 4c. It is clear that the growth rate increases with TMGa flow over the full range. The trend is not linear; however, for low TMGa (high V/ III), there is a steep increase, but at higher TMGa (low V/III) this trend slows, potentially saturating at very high TMGa. The apparently linear trend between growth rate and TMGa for low TMGa flow is consistent with our interpretation above that the (effectively) constant $\mathrm{Ga}$ concentration in the catalyst is a consequence of $\mathrm{Ga}$ limiting the growth rate. Following the reasoning of Mårtensson et al. ${ }^{64}$, we conclude that when $\mathrm{AsH}_{3}$ is very much in excess, the As concentration in the catalyst quickly reaches a maximum concentration which is in steady state with re-evaporation to the vapor; so long as the nucleation barrier does not shift significantly with growth parameters, the growth rate is limited by the time required for the $\mathrm{Ga}$ concentration to reach the level needed to overcome the nucleation barrier. The weakening of this trend at high TMGa, where increased $\mathrm{Ga}$ is observed in the catalyst, indicates a transition to a growth regime where As plays a limiting role. In this regime, the high TMGa flow allows the Ga concentration to exceed the value reached in the high V/III regime, before the As reaches the concentration that would be in steady state with vapor. Since the supersaturation is determined by both $\mathrm{Ga}$ and As species, the nucleation barrier is then reached for higher $\mathrm{Ga}$ and lower As concentrations (determined by the V/III ratio). The growth rate will then depend on both TMGa and $\mathrm{AsH}_{3}$ flows. True As-limited growth would be predicted for even higher TMGa flows, although it is not clear whether such a regime could actually be reached in experiments.

In addition to the change of the droplet size with V/III ratio, a change in the crystal structure of the nanowire was also observed. In the high V/III ratio regime ( 2500-5000), the nanowire grew in the wurtzite structure along the $\langle 0001\rangle$ direction (Fig. 5a, c). When the V/III ratio was decreased, stacking faults started to appear in the wurtzite nanowire. At even lower V/III ratios close to 1000 , the nanowire grew as a mixture of both zincblende and wurtzite structures. At still lower V/III ratio of $\sim 450$, the same nanowire grew in the zincblende structure along the $\langle 111\rangle$ axis (Fig. 5b, d). The change of nanowire structure from zincblende to wurtzite with increasing V/III has been reported experimentally 52,69 and 

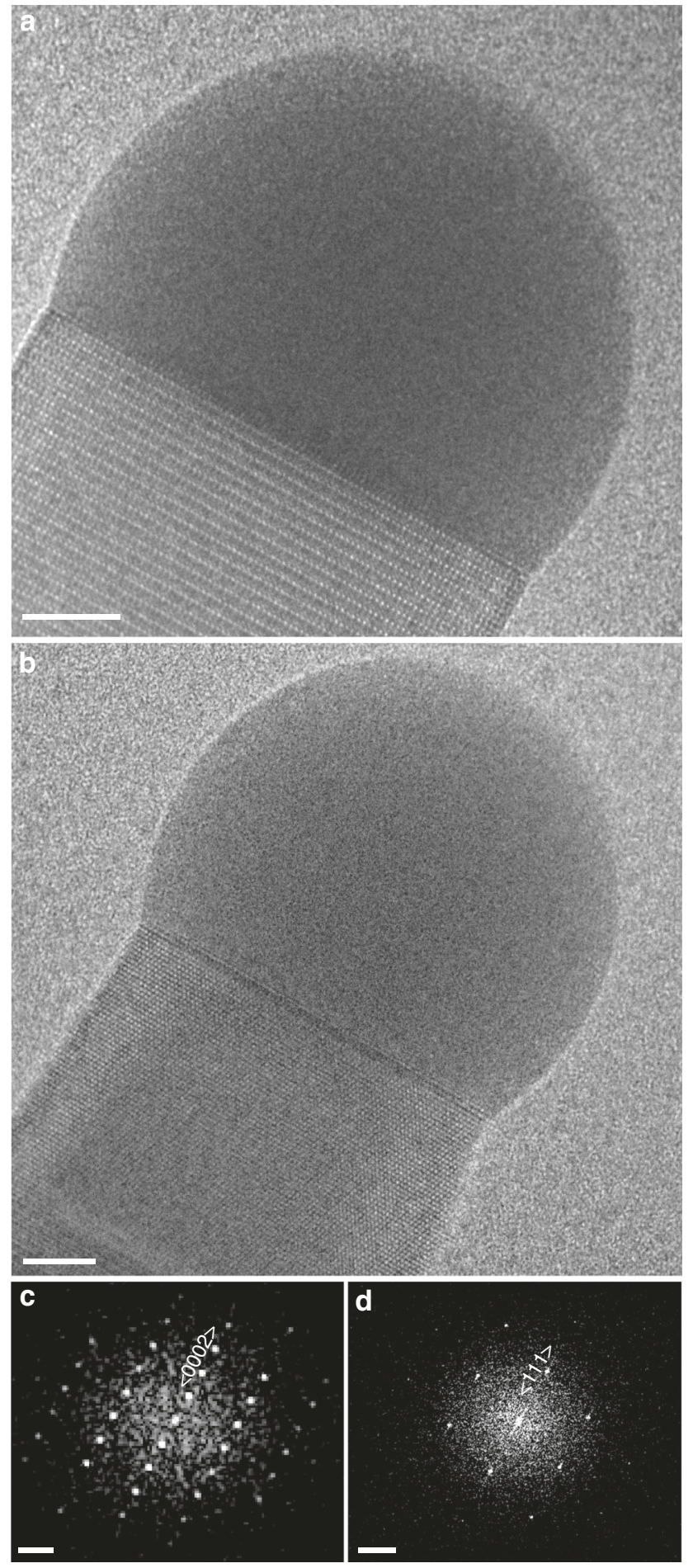

Fig. 5 Catalyst morphology and nanowire structure. a TEM image at high $\mathrm{V} / \mathrm{III}$ ratios where the nanowire is growing in the wurtzite structure. $\mathbf{b}$ TEM image at low $\mathrm{V} / \mathrm{III}$ ratios where the nanowire is growing in the zincblende structure. $\mathbf{c}, \mathbf{d}$ are processed reduced FFTs of panels $\mathbf{a}$ and $\mathbf{b}$, respectively, showing wurtzite and zincblende structure, respectively. Scale bars in panels $\mathbf{a}$ and $\mathbf{b}$ denote $5 \mathrm{~nm}$. Scale bars in panels $\mathbf{c}$ and $\mathbf{d}$ indicate $5 \mathrm{~nm}^{-1}$

theoretically ${ }^{24}$. MOCVD experiments also show a second transition back to zincblende at very high V/III ratio ${ }^{64,69}$, but this regime was not covered in the experiments described here. We have also observed in other experiments that, for even higher TMGa flows than studied here, the interface develops an oscillating truncation consistent with earlier in situ observations ${ }^{49-52}$. Since the interface dynamics are qualitatively very different in that regime, it was not covered during the experiments that are included in this study.

\section{Summary and outlook}

In summary, GaAs nanowires were grown with a gold-based catalyst particle in an environmental TEM in order to deepen the understanding of nanowire growth. The chemical composition of the catalyst particle was measured in situ as the nanowire was growing. We report the catalyst composition during growth of one nanowire as a function of temperature and another wire of similar dimensions as a function of ratio of precursor fluxes. Since the Ga concentration in the Ga-limited growth regime seem to be determined by thermodynamics, the results would be applicable to Au-catalyzed growth of GaAs nanowires independent of the growth method. Although the As content in the catalyst is close to the detection limit by XEDS, we can estimate for the As concentration a lower bound (by comparing measured $\mathrm{Ga}-\mathrm{Au}$ content with calculated $\mathrm{Au}-\mathrm{Ga}-\mathrm{As}$ phase diagrams) and an upper bound (from XEDS). The Ga concentration in the catalyst increases with increasing Ga precursor flux. These in situ measurements will aid better theoretical modeling of nanowire growth and improve the understanding of nanowire growth mechanisms. The precursor partial pressures and the nanowire growth rates in these experiments are similar to that in a typical ex situ MOCVD, which is why we believe the quantitative results will be applicable to the latter. The results could be extended at least qualitatively to other growth techniques as well. Most metalassisted III-V and II-VI nanowire growths typically have low solubility of the anion in the catalyst, and the Au-assisted GaAs studied here serves as a model system.

\section{Methods}

In situ nanowire growth. Gold aerosol particles of 30 -nm diameter on an average were used to seed nanowire growth. Silicon nitride MEMS heating chips from Norcada were used as the substrate. The thinnest $\operatorname{SiN}_{x}$ parts where growth was monitored had a thickness of $\sim 35 \mathrm{~nm}$. Atomic-resolved imaging was performed with an AMT XR401 sCMOS camera, and the videos were recorded at about $20 \mathrm{fps}$. The TEM images in the article were extracted from these videos and processed.

GaAs nanowires were grown in a Hitachi HF3300S environmental transmission electron microscope (ETEM) with CEOS B-COR-aberrationcorrector and a cold-field emission gun. Blaze software by Hitachi was used to control the local sample temperature using Joule heating in a constant power mode. The chips are calibrated to the melting point of gold with accuracy of $\pm 5^{\circ} \mathrm{C}$ by the manufacturer. The ETEM was connected to a gas handling system with the CVD gases. A single-tilt holder that has two separate microtubes running to the holder tip was used for supplying gases. The holder and the gashandling system are connected by a polymer-coated thin quartz tube (PEEKSil) from Trajan Scientific.

XEDS. The XEDS measurements were performed with an SDD X-Max ${ }^{N} 80 \mathrm{~T}$ system from Oxford Instruments. While measuring the catalyst composition, a small condenser aperture was used and the beam was condensed on the anterior part of the catalyst (opposite to the nanowire/catalyst interface). As the nanowire grows, the catalyst particle keeps moving forward. During XEDS, the illuminated area on the fluorescent screen was continuously monitored and the sample moved appropriately. Electron dose was in the order of 20,000 electrons $\AA^{-2} \mathrm{~s}^{-1}$ during XEDS. We specify the percentage content of the elements in terms of atomic percentage (and not as weight percentage) throughout this paper. The data were acquired and quantified with Aztec software. Acquisition was typically for $4 \mathrm{~min}$. In XEDS scans, we observe signals of $\mathrm{Au}, \mathrm{Ga}$, and As from the catalyst and/or nanowire, $\mathrm{Si}$ and $\mathrm{N}$ from the substrate and $\mathrm{Cu}$ due to scattering from parts of the microscope. The quantification result is renormalized such that $\mathrm{Au}+\mathrm{Ga}=100$ atomic \% to obtain the results quoted in this article, unless otherwise specified. The default lines ( $\mathrm{K}$ for $\mathrm{Ga}, \mathrm{L}$ for $\mathrm{Au}, \mathrm{K}$ for $\mathrm{As}$ ) were used for quantification. The standard deviation of quantification result from Aztec is shown as the $y$-axis error in Figs. $2 \mathrm{~b}$ and $4 \mathrm{~b}$. The background subtraction of XEDS spectrum is done by filtered least-squares fitting. More details about the XEDS quantification results are given in Supplementary Discussion 6. In all the plots in this article indicating error 
bars, the value is indicated as mean \pm SD. The reliability of the XEDS quantification was cross-checked by measuring stoichiometric GaAs with the same instrument and quantification tools.

Arsenic content from XEDS. Typically, the measured As signal is very low, and a clear peak cannot necessarily be distinguished from background. Full quantification of spectra (including background and artefacts such as $\mathrm{Si}, \mathrm{N}$, etc.) yields an As weight $\%$ of less than one, which is close to the detection limit in XEDS. Moreover, any small As signal detected may not necessarily originate from the catalyst nanoparticle. Since the nanowire is growing during the XEDS acquisition, the catalyst/nanowire interface moves. In order to track this motion manually, we try to not condense the beam to a spot, but slightly spread it so that a small portion at the front rim of the catalyst is visible. Imperfections in tracking the motion of the particle can contribute to XEDS signal from the nanowire part. This along with electrons scattered into the nanowire from the catalyst and also the electron in the periphery of the direct beam could easily lead to an overestimate of both Ga and As by a few percent.

Temperature series experiment. In the temperature series experiment discussed, temperature was increased from 440 to $500^{\circ} \mathrm{C}$ in steps of $20^{\circ} \mathrm{C}$, and finally decreased to $420^{\circ} \mathrm{C}$. After each temperature was reached, we waited at least a minute so that the catalyst stabilizes and there is no evident change in its dimensions. This wire grew in the wurtzite structure in the $\langle 0001\rangle$ direction at the conditions studied.

Phase diagrams shown here are calculated with the Thermo-Calc software using the thermodynamic data assessed by Ghasemi et al. ${ }^{66}$.

V/III series experiment. The measurement for the V/III series were recorded at $420^{\circ} \mathrm{C}$. We started to observe the nanowire when the V/III was 4500 , where we measured the first XEDS data. Then the Ga supply was stopped for some time. The TMGa flow was restarted, and increased slowly in steps until (at very high flow) the nanowire changed direction and folded back on itself during an XEDS acquisition. During the XEDS measurement at the lowest V/III ratio (447), there was some As signal observed due to scattering from GaAs NW, so the same percentage of Ga was subtracted from the quantification results and renormalized to obtain the data point plotted (see Supplementary Discussion 6 for details). The entire range (not SD) about which V/III varied during individual XEDS spectrum acquisition is denoted by the error bar for the $x$-axis in Fig. 4b. Each point in the plot of growth rate (Fig. 4c) is one measurement of the average growth rate of the nanowire in a particular time interval. From two different frames of the recorded videos, the time difference between the frames and the length the wire has grown in that time interval is noted, and growth rate calculated. The time difference we chose was typically $10-20 \mathrm{~s}$. In this interval, the variation/fluctuation in the precursor flow is less than a percent. So the error bars on the $x$-axis will be smaller than the symbols used.

Pressure at the sample. During growth experiments, the pressure near the pole piece was measured by an Inficon MPG400 pressure gauge, and is referred to as "column pressure" here. The precursor inlet tubes run along the length of the TEM holder, and precursor gases are released close to the heated $\mathrm{SiN}_{x}$ grid. Hence, the pressures are higher at the growth front than the "column pressure". The sample pressure relative to the column pressure was calibrated using the pressure at the heating coil of a clean $\mathrm{SiN}_{x}$ grid (without $\mathrm{Au}$ or GaAs) as a pressure gauge following the Pirani gauge principles using the Blaze software. We performed calibration experiments with $\mathrm{N}_{2}$ and also $\mathrm{H}_{2}$ and found that the pressure at the sample (measured by Blaze) is twice of "column pressure". A factor of two is therefore used to estimate sample pressure for each species based on its calibrated column pressure.

Precursor partial pressures and $\mathbf{V} / \mathrm{III}$ ratio. The TMGa bubbler was maintained at $-10{ }^{\circ} \mathrm{C}$ with $\mathrm{H}_{2}$ bubbled through it. In addition, a small fixed amount of $\mathrm{H}_{2}$ dilution (to be more precise, four times the flow used for the bubbler) was added in the TMGa line followed the bubbler. No additional carrier gas was used, and the $\mathrm{H}_{2}$ partial pressure is thus much lower than in a typical MOCVD. The flow of the $\mathrm{TMGa} / \mathrm{H}_{2}$ mixture was controlled by a mass flow controller (MFC), and a portion of the resulting flow was bypassed to the vent line to restrict the TMGa pressure reaching the microscope.

In order to determine the partial pressure of TMGa at the sample, the precursor fluxes sent to the ETEM were monitored with a residual gas analyzer (SRS RGA 300) using mass spectrometry in these experiments. The amount of the dominant TMGa derivatives (containing Ga) are measured at mass-to-charge ratio of 101, 99, 71 , and 69 associated with dimethylgallium and Ga. (The sample heating is very local at the $\mathrm{SiN}_{x}$ grid, decomposing just a very small fraction of the supplied precursors and so these RGA measurements are independent of localized pyrolysis at the sample.) Calibration experiments were performed for different but known TMGa and $\mathrm{H}_{2}$ flows to find the correlation between "column pressure" and the Garelated mass spectrometry reading at mass-to-charge ratio of 101 . We assume that
TMGa and $\mathrm{H}_{2}$ are being pumped out from the ETEM at the same efficiency, which might not be true, and hence the TMGa partial pressure quoted in this report could be an underestimate. TMGa partial pressure at the sample during experiments is therefore determined using the calibrated RGA readings together with the factor of two between column pressure and sample pressure described above.

The $\mathrm{AsH}_{3}$ flow was controlled exclusively by MFC (no part bypassed) and fixed for all experiments reported here at $\sim 1 \mathrm{~Pa}$ at the sample. V/III ratio is then calculated using this value divided by the TMGa pressure calculated using RGA readings for the specific experiment.

Inficon MPG400 pressure gauge, that was used during growth experiments, is gas dependent. So using a gas-independent capacitance pressure gauge $\mathrm{SKY}^{R}$ CDG045D, we found the correction factors for $\mathrm{H}_{2}, \mathrm{~N}_{2}$, and $\mathrm{AsH}_{3}$ in the appropriate pressure ranges. These correction factors were included in the precursor partial pressure calibration curves.

\section{Data availability}

More data that supports the findings of this study are available from the corresponding author upon request.

Received: 22 March 2019; Accepted: 10 September 2019; Published online: 08 October 2019

\section{References}

1. Guo, Y. N. et al. Structural characteristics of GaSb/GaAs nanowire heterostructures grown by metal-organic chemical vapor deposition. Appl. Phys. Lett. 89, 231917 (2006).

2. Larsson, M. W. et al. Strain mapping in free-standing heterostructured wurtzite InAs/InP nanowires. Nanotechnology 18, 015504 (2006)

3. Caroff, P. et al. InSb heterostructure nanowires: MOVPE growth under extreme lattice mismatch. Nanotechnology 20, 495606 (2009).

4. Persson, A. I. et al. Solid-phase diffusion mechanism for GaAs nanowire growth. Nat. Mater. 3, 677-681 (2004).

5. Jacobs, B. W. et al. Electronic and structural characteristics of zinc-blende wurtzite biphasic homostructure GaN nanowires. Nano Lett. 7, 1435-1438 (2007).

6. Joyce, H. J., Wong-Leung, J., Gao, Q., Tan, H. H. \& Jagadish, C. Phase perfection in zinc blende and wurtzite III-V nanowires using basic growth parameters. Nano Lett. 10, 908 (2010).

7. Dick, K. A. et al. Control of III-V nanowire crystal structure by growth parameter tuning. Semiconductor Sci. Technol. 25, 024009 (2010).

8. Sukrittanon, $\mathrm{S}$. et al. Growth and characterization of dilute nitride $\mathrm{GaN}_{x} \mathrm{P}_{1-x}$ nanowires and $\mathrm{GaN}_{x} \mathrm{P}_{1-x} / \mathrm{GaN}_{y} \mathrm{P}_{1-y}$ core/shell nanowires on $\mathrm{Si}$ (111) by gas source molecular beam epitaxy. Appl. Phys. Lett. 105, 072107 (2014).

9. Namazi, L., Ghalamestani, S. G., Lehmann, S., Zamani, R. R. \& Dick, K. A. Direct nucleation, morphology and compositional tuning of InAs ${ }_{1-x} \mathrm{Sb}_{x}$ nanowires on InAs (111) B substrates. Nanotechnology 28, 165601 (2017).

10. Dubrovskii, V. G. Refinement of nucleation theory for vaporliquidsolid nanowires. Cryst. Growth Des. 17, 2589-2593 (2017).

11. Schwarz, K. W. \& Tersoff, J. Elementary processes in nanowire growth. Nano Lett. 11, 316-320 (2011).

12. Nebol'sin, V. A. \& Shchetinin, A. A. Role of surface energy in the vapor-liquid-solid growth of silicon. Inorg. Mater. 39, 899-903 (2003).

13. Krogstrup, P. et al. Advances in the theory of III-V nanowire growth dynamics. J. Phys. D: Appl. Phys. 46, 313001 (2013).

14. Glas, F. Chemical potentials for Au-assisted vapor-liquid-solid growth of III-V nanowires. J. Appl. Phys. 108, 073506 (2010).

15. Dubrovskii, V. G. Group V sensitive vapor-liquid-solid growth of Aucatalyzed and self-catalyzed III-V nanowires. J. Cryst. Growth 440, 62-68 (2016).

16. Plante, M. C. \& LaPierre, R. R. Analytical description of the metal-assisted growth of III-V nanowires: axial and radial growths. J. Appl. Phys. 105, 114304 (2009).

17. Ramdani, M. R., Harmand, J. C., Glas, F., Patriarche, G. \& Travers, L. Arsenic pathways in self-catalyzed growth of GaAs nanowires. Cryst. Growth Des. 13, 91-96 (2013).

18. Dubrovskii, V. G. et al. Diffusion-controlled growth of semiconductor nanowires: vapor pressure versus high vacuum deposition. Surf. Sci. 601, 4395-4401 (2007)

19. Glas, F., Harmand, J. C. \& Patriarche, G. Why does wurtzite form in nanowires of III-V zinc blende semiconductors? Phys. Rev. Lett. 99, 146101 (2007).

20. Dubrovskii, V. G., Sibirev, N. V., Harmand, J. C. \& Glas, F. Growth kinetics and crystal structure of semiconductor nanowires. Phys. Rev. B 78, 235301 (2008). 
21. Johansson, J., Zanolli, Z. \& Dick, K. A. Polytype attainability in III-V semiconductor nanowires. Cryst. growth Des. 16, 371-379 (2015).

22. Tornberg, M., Dick, K. A. \& Lehmann, S. Thermodynamic stability of goldassisted InAs nanowire growth. J. Phys. Chem. C 121, 21678-21684 (2017).

23. Panciera, F. et al. Controlling nanowire growth through electric field-induced deformation of the catalyst droplet. Nat. Commun. 7, 12271 (2016).

24. Dubrovskii, V. G. Mono- and polynucleation, atomistic growth, and crystal phase of III-V nanowires under varying group V flow. J. Chem. Phys. 142, 204702 (2015)

25. Krogstrup, P. et al. Impact of the liquid phase shape on the structure of III-V nanowires. Phys. Rev. Lett. 106, 125505 (2011).

26. Algra, R. E. et al. Formation of wurtzite InP nanowires explained by liquidordering. Nano Lett. 11, 44-48 (2011).

27. Dubrovskii, V. G. \& Sibirev, N. V. General form of the dependences of nanowire growth rate on the nanowire radius. J. Cryst. growth 304, 504-513 (2007).

28. Fröberg, L. E., Seifert, W. \& Johansson, J. Diameter-dependent growth rate of InAs nanowires. Phys. Rev. B 76, 153401 (2007).

29. Li, N., Li, W., Liu, L. \& Tan, T. Y. A nucleation-growth model of nanowires produced by the vapor-liquid-solid process. J. Appl. Phys. 114, 064302 (2013).

30. Borgström, M. T., Immink, G., Ketelaars, B., Algra, R. \& Bakkers, E. P. A. M. Synergetic nanowire growth. Nat. Nanotechnol. 2, 541 (2007).

31. Li, A., Sibirev, N. V., Ercolani, D., Dubrovskii, V. G. \& Sorba, L. Readsorption assisted growth of InAs/InSb heterostructured nanowire arrays. Cryst. Growth Des. 13, 878-882 (2013).

32. Borg, B. M., Johansson, J., Storm, K. \& Deppert, K. Geometric model for metalorganic vapour phase epitaxy of dense nanowire arrays. J. Cryst. Growth 366, 15-19 (2013).

33. Ek, M. \& Filler, M. A. Atomic-scale choreography of vapor-liquid-solid nanowire growth. Acc. Chem. Res. 51, 118-126 (2018).

34. Kirkham, M., Wang, Z. L. \& Snyder, R. L. Tracking the catalyzed growth process of nanowires by in situ x-ray diffraction. J. Appl. Phys. 108, 014304 (2010).

35. Schroth, P. et al. Evolution of polytypism in GaAs nanowires during growth revealed by time-resolved in situ x-ray diffraction. Phys. Rev. Lett. 114, 055504 (2015).

36. Krogstrup, P. et al. In-situ x-ray characterization of wurtzite formation in GaAs nanowires. Appl. Phys. Lett. 100, 093103 (2012).

37. Sivaram, S. V., Hui, H. Y., de la Mata, M., Arbiol, J. \& Filler, M. A. Surface hydrogen enables subeutectic vapor-liquid-solid semiconductor nanowire growth. Nano Lett. 16, 6717-6723 (2016).

38. Sivaram, S. V., Shin, N., Chou, L. W. \& Filler, M. A. Direct observation of transient surface species during Ge nanowire growth and their influence on growth stability. J. Am. Chem. Soc. 137, 9861-9869 (2015).

39. Shin, N., Chi, M. \& Filler, M. A. Interplay between defect propagation and surface hydrogen in silicon nanowire kinking superstructures. ACS nano $\mathbf{8}$, 3829-3835 (2014).

40. Tchernycheva, M., Harmand, J. C., Patriarche, G., Travers, L. \& Cirlin, G. E. Temperature conditions for GaAs nanowire formation by Au-assisted molecular beam epitaxy. Nanotechnology 17, 4025-4030 (2006).

41. $\mathrm{Xu}, \mathrm{T}$. et al. Faceting, composition and crystal phase evolution in III-V antimonide nanowire heterostructures revealed by combining microscopy techniques. Nanotechnology 23, 095702 (2012).

42. Jo, J., Tchoe, Y., Yi, G. C. \& Kim, M. Real-time characterization using in situ RHEED transmission mode and TEM for investigation of the growth behaviour of nanomaterials. Sci. Rep. 8, 1694 (2018).

43. Heurlin, M., Anttu, N., Camus, C., Samuelson, L. \& Borgström, M. T. In situ characterization of nanowire dimensions and growth dynamics by optical reflectance. Nano Lett. 15, 3597-3602 (2015).

44. Clement, T., Ingole, S., Ketharanathan, S., Drucker, J. \& Picraux, S. T. In situ studies of semiconductor nanowire growth using optical reflectometry. Appl. Phys. Lett. 89, 163125 (2006)

45. Fernández-Garrido, S., Zettler, J. K., Geelhaar, L. \& Brandt, O. Monitoring the formation of nanowires by line-of-sight quadrupole mass spectrometry: a comprehensive description of the temporal evolution of gan nanowire ensembles. Nano Lett. 15, 1930-1937 (2015).

46. Kolíbal, M., Pejchal, T., Vystavel, T. \& Sikola, T. The synergic effect of atomic hydrogen adsorption and catalyst spreading on Ge nanowire growth orientation and kinking. Nano Lett. 16, 4880-4886 (2016).

47. Kodambaka, S., Tersoff, J., Reuter, M. C. \& Ross, F. M. Germanium nanowire growth below the eutectic temperature. Science 316, 729-732 (2007).

48. Hofmann, S. et al. Ledge-flow-controlled catalyst interface dynamics during $\mathrm{Si}$ nanowire growth. Nat. Mater. 7, 372-375 (2008)

49. Wen, C.-Y. et al. Periodically changing morphology of the growth interface in Si, Ge, and GaP nanowires. Phys. Rev. Lett. 107, 025503 (2011).
50. Gamalski, A. D., Ducati, C. \& Hofmann, S. Cyclic supersaturation and triple phase boundary dynamics in germanium nanowire growth. J. Phys. Chem. C. 115, 4413-4417 (2011).

51. Chou, Y.-C. et al. Atomic-scale variability and control of III-V nanowire growth kinetics. Science 343, 281-284 (2014).

52. Jacobsson, D. et al. Interface dynamics and crystal phase switching in GaAs nanowires. Nature 531, 317-322 (2016).

53. Harmand, J. C. et al. Atomic step flow on a nanofacet. Phys. Rev. Lett. 121, 166101 (2018)

54. Gamalski, A. D., Tersoff, J. \& Stach, E. A. Atomic resolution in situ imaging of a double-bilayer multistep growth mode in gallium nitride nanowires. Nano Lett. 16, 2283-2288 (2016).

55. Dubrovskii, V. G., Sokolova, Z. V., Rylkova, M. V. \& Zhiglinsky, A. A. Composition and contact angle of Au-III-V droplets on top of Au-catalyzed III-V nanowires. Mater. Phys. Mech. 36, 1-7 (2018).

56. Dubrovskii, V. G. \& Grecenkov, J. Zeldovich nucleation rate, self-consistency renormalization, and crystal phase of Au-catalyzed GaAs nanowires. Cryst. Growth Des. 15, 340-347 (2015).

57. Dubrovskii, V. G. Influence of the group V element on the chemical potential and crystal structure of Au-catalyzed III-V nanowires. Appl. Phys. Lett. 104, 053110 (2014)

58. Leshchenko, E. D., Ghasemi, M., Dubrovskii, V. G. \& Johansson, J. Nucleation-limited composition of ternary III-V nanowires forming from quaternary gold based liquid alloys. CrystEngComm 20, 1649-1655 (2018).

59. Harmand, J. C. et al. Analysis of vapor-liquid-solid mechanism in Au-assisted GaAs nanowire growth. Appl. Phys. Lett. 87, 203101 (2005).

60. Jacobsson, D., Lehmann, S. \& Dick, K. A. Zincblende-to-wurtzite interface improvement by group III loading in Au-seeded GaAs nanowires. Phys. status solidi (RRL) Rapid Res. Lett. 7, 855-859 (2013).

61. Tersoff, J. Stable self-catalyzed growth of III-V nanowires. Nano Lett. 15, 6609-6613 (2015)

62. Tizei, L. H. G., Chiaramonte, T., Ugarte, D. \& Cotta, M. A. III-V semiconductor nanowire growth: does arsenic diffuse through the metal nanoparticle catalyst. Nanotechnology 20, 275604 (2009)

63. Glas, F., Ramdani, M. R., Patriarche, G. \& Harmand, J. C. Predictive modeling of self-catalyzed III-V nanowire growth. Phys. Rev. B 88, 195304 (2013).

64. Mårtensson, E. K., Lehmann, S., Dick, K. A. \& Johansson, J. Simulation of GaAs nanowire growth and crystal structure. Nano Lett. 19, 1197-1203 (2019).

65. Chatillon, C., Hodaj, F. \& Pisch, A. Thermodynamics of GaAs nanowire MBE growth with gold droplets. J. Cryst. Growth 311, 3598-3608 (2009).

66. Ghasemi, M. \& Johansson, J. Phase diagrams for understanding gold-seeded growth of GaAs and InAs nanowires. J. Phys. D: Appl. Phys. 50, 134002 (2017)

67. Maliakkal, C. B. et al. Step-flow growth of III-V nanowire layers. Preprint at: https://arxiv.org/abs/1905.08225 (2019).

68. Tornberg, M. et al. Kinetics of Au-Ga droplet mediated decomposition of GaAs Nanowires. Nano Lett. 19, 3498-3504 (2019).

69. Lehmann, S., Jacobsson, D. \& Dick, K. A. Crystal phase control in GaAs nanowires: opposing trends in the $\mathrm{Ga}$-and As-limited growth regimes. Nanotechnology 26, 301001 (2015).

70. Dheeraj, D. L. et al. Controlling crystal phases in GaAs nanowires grown by Au-assisted molecular beam epitaxy. Nanotechnology 24, 015601 (2012).

71. Dubrovskii, V. G., Sokolova, Z. V., Rylkova, M. V. \& Zhiglinsky, A. A. Composition and contact angle of Au-III-V droplets on top of Au-C III-V nanowires. Mater. Phys. Mech. 36, 1-7 (2018).

\section{Acknowledgements}

We wish to acknowledge support from the Knut and Alice Wallenberg Foundation, NanoLund, and the Swedish Research Council. We thank E. Mårtensson for valuable discussions and insights. We are grateful to Joacim Gustafsson, Stas Dogel, and Charles Soong from Hitachi High-Technologies and John Wheeler and Shaun Ohman from Collabratech for technical assistance in setting up the laboratory. We thank Robin Sjökvist for assistance during the precursor flow calibration experiments. Open access funding provided by Lund University.

\section{Author contributions}

C.B.M., D.J., M.T. and A.R.P. performed the experiments. Data analysis was done by C.B. M. J.J. performed the phase diagram calculations. K.A.D. and R.W. coordinated the project. All authors discussed the results and contributed to the concepts discussed in this article.

\section{Competing interests}

The authors declare no competing interests. 


\section{Additional information}

Supplementary information is available for this paper at https://doi.org/10.1038/s41467019-12437-6.

Correspondence and requests for materials should be addressed to C.B.M.

Peer review information Nature Communications thanks Sonia Conesa-Boj and the other, anonymous, reviewer(s) for their contribution to the peer review of this work. Peer reviewer reports are available.

Reprints and permission information is available at http://www.nature.com/reprints

Publisher's note Springer Nature remains neutral with regard to jurisdictional claims in published maps and institutional affiliations. (c) (i) Open Access This article is licensed under a Creative Commons Attribution 4.0 International License, which permits use, sharing, adaptation, distribution and reproduction in any medium or format, as long as you give appropriate credit to the original author(s) and the source, provide a link to the Creative Commons license, and indicate if changes were made. The images or other third party material in this article are included in the article's Creative Commons license, unless indicated otherwise in a credit line to the material. If material is not included in the article's Creative Commons license and your intended use is not permitted by statutory regulation or exceeds the permitted use, you will need to obtain permission directly from the copyright holder. To view a copy of this license, visit http://creativecommons.org/ licenses/by/4.0/.

(C) The Author(s) 2019 